Degradable magnesium implant-associated infections by bacterial biofilms induce robust localized and systemic inflammatory reactions in a mouse model

This content has been downloaded from IOPscience. Please scroll down to see the full text.

Download details:

IP Address: 146.107.23.26

This content was downloaded on 28/06/2017 at 13:29

Manuscript version: Accepted Manuscript

Rahim et al

To cite this article before publication: Rahim et al, 2017, Biomed. Mater., at press:

https://doi.org/10.1088/1748-605X/aa7667

This Accepted Manuscript is: (c) 2017 IOP Publishing Ltd

During the embargo period (the 12 month period from the publication of the Version of Record of this article), the Accepted Manuscript is fully protected by copyright and cannot be reused or reposted elsewhere.

As the Version of Record of this article is going to be / has been published on a subscription basis, this Accepted Manuscript is available for reuse under a CC BY-NC-ND 3.0 licence after the 12 month embargo period.

After the embargo period, everyone is permitted to copy and redistribute this article for non-commercial purposes only, provided that they adhere to all the terms of the licence https://creativecommons.org/licences/by-nc-nd/3.0

Although reasonable endeavours have been taken to obtain all necessary permissions from third parties to include their copyrighted content within this article, their full citation and copyright line may not be present in this Accepted Manuscript version. Before using any content from this article, please refer to the Version of Record on IOPscience once published for full citation and copyright details, as permission will likely be required. All third party content is fully copyright protected, unless specifically stated otherwise in the figure caption in the Version of Record.

When available, you can view the Version of Record for this article at: http://iopscience.iop.org/article/10.1088/1748-605X/aa7667 


\section{Degradable magnesium implant-associated infections by bacterial biofilms induce robust localized and systemic inflammatory reactions in a mouse model} Muhammad Imran Rahim*1,3, Anshu Babbar ${ }^{1}$, Stefan Lienenklaus ${ }^{2}$, Marina C. Pils ${ }^{1}$, Manfred Rohde ${ }^{1}$

${ }^{1}$ Central Facility for Microscopy, Animal Experimental Unit, Helmholtz Centre for Infection Research, Inhoffenstrasse 7, 38124 Braunschweig, Germany

2Institute for Laboratory Animal Science, Hannover Medical School, Carl-Neuberg-

Strasse 1, 30625 Hannover, Germany and Institute for Experimental Infection Research, TWINCORE, Centre for Experimental and Clinical Infection Research, Feodor-Lynen-

Strasse 7, 30625 Hannover, Germany

${ }^{3}$ Present address: Klinik für Zahnärztliche Prothetik und Biomedizinische

Werkstoffkunde, Hannover Medical School, Carl-Neuberg-Strasse 1, 30625 Hannover, Germany

*Address correspondence to this author at Klinik für Zahnärztliche Prothetik und Biomedizinische Werkstoffkunde, Hannover Medical School, Carl-Neuberg-Strasse 1, 30625 Hannover, Germany Tel: +49 (0)511-5321431

E-mail: Rahim.Muhammad@mh-hannover.de 


\section{Abstract}

Biomaterial-associated Pseudomonas aeruginosa biofilm infections constitute cascade of host immune reactions ultimately leading towards implant failure. Due to lack of relevant in vivo biofilm models, majority of the studies report host immune responses against free living or planktonic bacteria while bacteria in clinical situations live more frequently as biofilm communities than as single cells. Present study investigated host immune responses against biomaterial-associated $P$. aeruginosa biofilms in a clinically relevant mouse model. Previously, we reported metallic magnesium, a prospective biodegradable implant, to be permissive for bacterial biofilms in vivo even though it exhibits antibacterial properties in vitro. Therefore, magnesium was employed as biomaterial to investigate in vivo biofilm formation and associated host immune responses by using two $P$. aeruginosa strains and two mouse strains. $P$. aeruginosa formed biofilms on subcutaneously implanted magnesium discs. Non-invasive in vivo imaging indicated transient inflammatory responses at control sites whereas robust prolonged interferon- $\beta$ (IFN- $\beta$ ) expression was observed from biofilms in a transgenic animal reporter. Further, immunohistology and electron microscopic results showed that bacterial biofilms were located in two dimensions immediately on the implant surface and at a short distance in the adjacent tissue. These biofilms were surrounded by inflammatory cells (mainly polymorphonuclear cells) as compared to controls. Interestingly, even though the number of live bacteria in various organs remained below detectable levels, splenomegaly indicated systemic inflammatory processes. Overall, these findings confirmed the resistance of biofilm infections in vivo to potentially antibacterial properties of magnesium degradation products. In vivo imaging and histology indicated the induction of both, local and systemic host inflammatory responses against $P$. aeruginosa biofilms. Even though the innate host immune defenses 
could not eliminate the local infection for up to two weeks, there was no apparent systemic bacteremia and all animals investigated survived the infection.

Keywords: Magnesium implants; Biofilms; Immune system; Interferon; Immunohistology; Mouse model 


\section{Introduction}

Applications of medical implants have increased manifold in humans for reasons such as the organs loss due to chronic diseases and accidents [1]. Pseudomonas aeruginosa is an principal pathogen responsible for biofilm formation on medical implants $[2,3]$. Biofilms are the most precarious infections since they are thousands of times more resistant against conventional antibiotics and host immune systems [4-6]. Biomaterialassociated biofilm infections are mostly treated by surgical removal of implant from infected tissue followed by administration of antibiotics [7]. Such inevitable surgery together with antibiotic administration incurs huge losses on/patient health and economy. Moreover, biofilm infections trigger unrestrained host immune reactions which can cause immunosuppression, shock, transudation in organs and defects in coagulation [8-10]. Innate and adaptive immune responses to various pathogens comprise of accumulating phagocytic cells and inflammatory cytokines, among others interferons (IFNs), play a critical role $[11,12]$. However, performance of host immune responses is compromised when most of the implant materials require a surgical procedure for their insertion into body $[13,14]$. Initially, open wounds, body liquidfilled gaps between the implant and the surrounding tissue, structural, mechanical and chemical properties of implants hinder immune cells from invading bacteria [15-19]. Later, bacteria secrete extracellular matrix material (ECM) which prevents phagocytic immune cells such as macrophages and neutrophilic granulocytes from engulfing bacteria. The ECM also limits the diffusion rate of nutrients and metabolites, thereby reducing the bacterial metabolic activities. This impedes the efficacy of proliferationdependent antibiotics thereby reducing efficacy of various antibiotics [20-22]. To counter biofilm infections and to augment the performance of implant materials, 
various strategies have been introduced. These include, metal alloy implants with antimicrobial properties, antibacterial as well as anti-adhesive nanoparticles coatings and localized antimicrobial drug delivery systems on implant surfaces [23, 24]. Metallic magnesium as biodegradable implant material is being highly investigated for clinical applications due to its in vitro bactericidal corrosion products and antibacterial properties which can efficiently counter biomaterial-associated biofilm infections [2528]. Magnesium alloys are presently under intense investigation as degradable, yet sturdy implant materials that could potentially be used for temporary applications to improve bone-healing and avoid long-term side effects associated with permanent metallic implants [29-31]. From our previous investigation as well as from literature, magnesium and its alloys are reported susceptible to $P$. aeruginosa colonization and subsequent biofilm formation [32-34]. Since, limited information exists on the capacity of biomaterial-associated P. aeruginosa biofilms to escape the local innate immune defense and spread into adjacent tissue or even systemically due to lack of clinically relevant biofilm mouse models $[35,36]$. In addition, there is no in vivo information about the role of interferons against biofilm infections. Therefore, we employed in vivo $P$. aeruginosa biofilm formation on magnesium as a mouse model and report here $P$. aeruginosa biofilm repercussions directly on the implant interface, in the adjacent tissue and systemically. We used nohinvasive in vivo imaging system to monitor in vivo biofilm formation and interferon response against these infections[37]. Side by side, morphological, histological and electron microscopic analyses were performed to determine biofilm effects on host.

\section{Material and Methods}

\subsection{Preparation of magnesium samples and bacterial cultures}


Disc shaped magnesium samples with (99.95\% purity) having $5 \mathrm{~mm}$ diameter and 2 $\mathrm{mm}$ thickness were prepared and processed as described previously [38]. Bioluminescent and wild type P. aeruginosa strains were streaked on LB agar plates and incubated for $18 \mathrm{~h}$ at $37{ }^{\circ} \mathrm{C}[39,40]$. For liquid cultures, isolates were cultured in $\mathrm{BL}$ broth medium and kept at $37^{\circ} \mathrm{C}$ with shaking speed of $150 \mathrm{RPM}$ till the desired $\mathrm{OD}_{600}=0.1$ was achieved. The bacterial suspension was then immediately placed on crushed wet ice to inhibit further growth.

\subsection{Surgical procedures and in vivo infections}

8 weeks old healthy female wild-type BALB/c mice were purchased (Harlan Winkelmann, Borchen, Germany) and IFN- $\beta$-reporter mice (IFN- $\beta^{+/ \Delta \beta-l u c}$ ) on the BALB/c background (ifnb1 $1^{\text {tm1.2Lien}) ~[41] ~ w e r e ~ b r e d ~ a t ~ t h e ~ H e l m h o l t z ~ C e n t r e ~ f o r ~ I n f e c t i o n ~}$ Research, Braunschweig. Mice were kept in individually ventilated cages in a dedicated infection facility with water and food ad libitum. Before surgery, animals were anesthetized with xylazine $(4 \mathrm{mg} / \mathrm{Kg})$ and ketamine $(10 \mathrm{mg} / \mathrm{Kg})$ and fur was removed at the site of surgery using a hair trimmer (Aesculap Suhl GmbH, Germany). Under sterile conditions in a laminar flow hood, $0.5 \mathrm{~cm}$ long incisions in the skin were made at three sites on the back of the mice using surgical scissors (Fine Science Tools GmbH, Heidelberg, Germany). A subcutaneous pouch was created from the incisions and 99.95 $\%$ pure magnesium discs with $5 \mathrm{~mm}$ diameter and $2 \mathrm{~mm}$ thickness were inserted [26]. The wounds were closed by applying simple interrupted sutures (Ethicon Vicryl, Johnson \& Johnson Medical GmbH). Immediately after implantation, logarithmically growing cultures of wild type $P$. aeruginosa (PA01) or bioluminescent $P$. aeruginosa (PA01 CTX::lux) at $\mathrm{OD}_{600}=0.1$ were injected subcutaneously onto the surface of implanted discs $[42,43]$. For monitoring the host interferon response, wild type $P$. 
aeruginosa (PA01) were injected on subcutaneously implanted magnesium discs in IFN$\beta$ reporter (IFN- $\beta^{+/ \Delta \beta-l u c}$ ) BALB $/ c$ mice, which allow tracking of IFN- $\beta$ gene induction [41]. D-luciferin (150 mg/Kg) (Calipers) in PBS was injected intraperitoneally $15 \mathrm{~min}$. before examination by in vivo imaging. In vivo luminescence was recorded by using a Xenogen IVIS-200 optical imaging system (Caliper Life Sciences) equipped with XGI-8 gas anesthesia unit (Caliper Life Sciences, Hopkinton, MA) using 2\% isoflurane. IFN- $\beta$ reporter mice were examined daily for a period of two weeks. BALB/c mice were euthanized on $8^{\text {th }}$ day post-infection and then magnesium implants with adjacent tissue were removed and fixed in a buffered formalin solution according to standard procedures [32]. Animal experiments were performed with the permission of the Lower Saxony State Office for Consumer Protection and Food Safety (LAVES; Oldenburg, Germany) under approval number 33.42502/07-10.5.

\subsection{Field emission scanning electron microscopy}

For electron microscopic examination, explanted magnesium and tissue samples were sputter coated with palladium-gold. Peri-implant tissues were fixed with 5\% formaldehyde and 2\% glutaraldehyde in HEPES buffer (100 mM HEPES, 90 mM sucrose, $10 \mathrm{mM} \mathrm{MgCl}_{2}, 10 \mathrm{mM} \mathrm{CaCl}_{2}, \mathrm{pH}$ 6.9) washed twice in TE buffer (10 mM TRIS, $2 \mathrm{mM}$ MEDTA, pH 6.9), dehydrated in a graded series of acetone $(10,30,50,70,90,100 \%)$ solutions for $15 \mathrm{~min}$ on ice and then dehydrated further in $100 \%$ acetone at room temperature. Subsequently, critical-point drying was performed with liquid $\mathrm{CO}_{2}$ (CPD300, Leica or CPD030 Bal-Tec). Dry tissue samples were sputter coated with palladium-gold (SCD500, Bal-Tec). Samples were examined using a Zeiss Merlin field emission scanning electron microscope at an acceleration of $5 \mathrm{kV}$ and by using the Zeiss SmartSEM software version 5.05 (Zeiss, Oberkochen, Germany). 


\subsection{Transmission electron microscopy}

Mice bearing infected implants were euthanized with $\mathrm{CO}_{2}$ followed by cervical dislocation. Peri-implant tissue was fixed in HEPES buffer containing 2.5\% glutaraldehyde and 5\% formaldehyde. The samples were washed with HEPES buffer and then incubated for one hour at room temperature in $1 \%$ aqueous osmium solution. Samples were dehydrated by serial incubation in acetone-water mixtures with increasing acetone concentrations $(10 \%, 30 \%$ and $50 \%)$ on ice, followed by a dehydration step in $70 \%$ acetone. Then the samples were incubated overnight in $70 \%$ acetone and $2 \%$ uranyl acetate at $4^{\circ} \mathrm{C}$. The following day, the dehydration process was continued in $90 \%$ and $100 \%$ acetone on ice and eventually in $100 \%$ acetone at room temperature. Samples were then embedded in epoxy resin as previously described [44]. Ultrathin sections from these samples were prepared with a diamond knife, picked up with butvar-coated grids, counterstained with uranyl acetate and lead citrate and examined in a TEM910 transmission electron microscope (Carl Zeiss, Oberkochen) set to an acceleration voltage of $80 \mathrm{kV}$. TEM images were digitally recorded at calibrated magnifications with a Slow-Scan CCD-Camera (ProScan, 1024x1024, Scheuring, Germany) using the ITEM-Software (Olympus Soft Imaging Solutions, Münster, Germany). Brightness and contrast were adjusted with the help of Adobe Photoshop version (Adobe).

\subsection{Histological procedures}

For histological analysis, animals were sacrificed by $\mathrm{CO}_{2}$ asphyxiation followed by cervical dislocation. Infected peri-implant tissues were isolated and fixed in $4 \%$ formalin for three days as previously described in detail [45]. Briefly, samples were dehydrated in a series of increasingly concentrated ethanol solutions and then 
embedded in paraffin. Histological slides of approximately $3 \mu \mathrm{m}$ thickness were prepared from paraffin embedded tissue samples. The sections were stained with Hematoxylin and Eosin (H\&E) or Periodic Acid Schiff (PAS) staining according to standard laboratory procedures. Moreover, immunohistology staining for Pseudomonas was performed with rabbit-polyclonal anti-Pseudomonas, (Biotrend, AP086) after heatmediated antigen retrieval. The samples were evaluated histo-pathologically on microscope slides using a light-microscope, randomized and blinded to the experimental groups.

\subsection{Tissue homogenization and colony forming unit assay}

One week after infection, mice bearing biofilms were peacefully euthanized with $\mathrm{CO}_{2}$ followed by cervical dislocation and their visceral cavities were opened. Visceral organs such as liver, spleen and gut were removed and their weights were measured. In parallel, subcutaneously implanted magnesium discs inundated with biofilms and the adjacent tissue mass were isolated. Liver, gut and peri-implant tissue were then shifted in homogenization tubes containing $0.1 \%(\mathrm{v} / \mathrm{v}) \mathrm{X}-100 / \mathrm{PBS}$ Triton solution. These tubes were then fixed in Polytron PT3000 homogenizer (Kinematica) and homogenization process was carried for 86 seconds. Explanted infected magnesium discs were directly submerged in PBS and vigorous vortexed for $5 \mathrm{~min}$. at max. speed to disrupt attached biofilm mass. Samples were analyzed for bacterial cell density (CFU/ml) by tenfold serial dilution and plating on LB plates kept at $37^{\circ} \mathrm{C}$ for $18 \mathrm{~h}$.

\section{Results}

\subsection{P. aeruginosa biofilm formation on magnesium implants in a mouse model}


A major challenge towards establishing a biofilm mouse model is rapid elimination of bacteria by host immune system. To establish a mouse model which supports biofilm infections for prolonged period of time, we implanted subcutaneously three magnesium discs at selected locations in a mouse and then injected bioluminescent $P$. aeruginosa (PA01) directly on each of these implants (Figure1, d0). In agreement with our previous findings, magnesium implants augmented the survival of bacteria on their surfaces [32]. Bacteria formed biofilms on magnesium and persisted for two weeks on the surface of these implants (Figure1, d0-d14). This experiment confirmed and reproduced our previous findings in that magnesium promotes biofilm formation for extended period of time. While, there is not a single mouse model which allows biofilm formation on metallic implants with plain surfaces in the presence of active host immune system. Therefore, we employed this system to investigate distribution of biofilm infections and associated host immune responses.

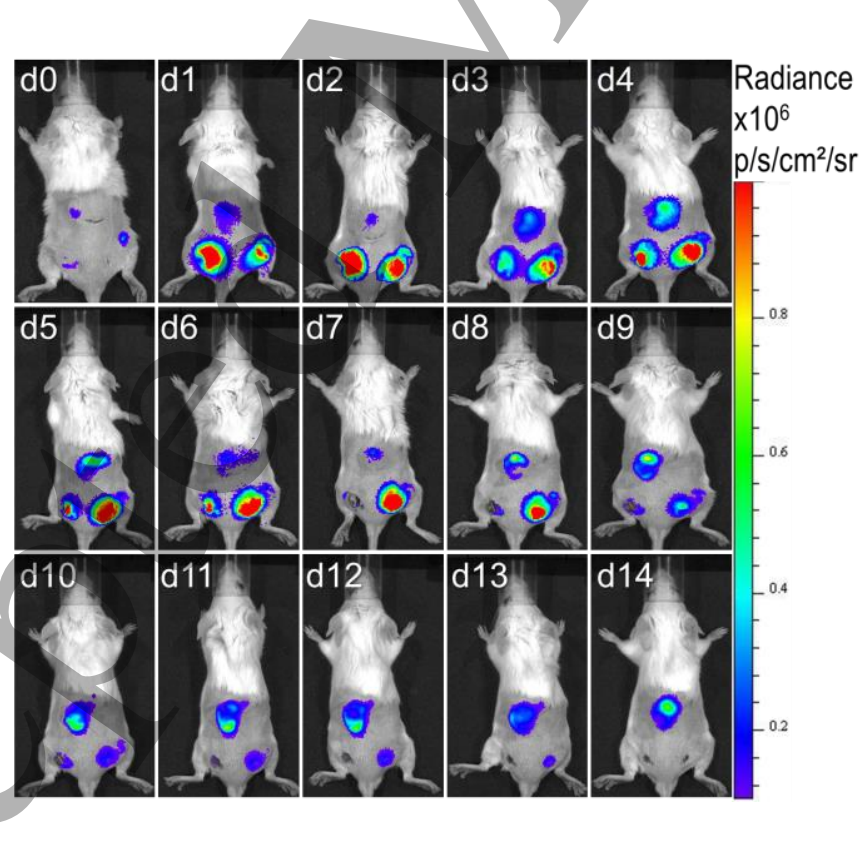

Figure 1: Magnesium implants are susceptible for $P$. aeruginosa biofilm formation. Magnesium discs were surgically inserted at three selected sites underneath the skin in mouse. Immediately after implantation, $5 \mu \mathrm{l}$ of bioluminescent $P$. aeruginosa at $\mathrm{OD}_{600}=0.1$ were injected (d0, color spots) on each of the implant. Mouse was then observed under in vivo imager (d0) and then on daily basis for two weeks. Luminescent spots indicate bacterial biofilm formation over the indicated time points in days (d). 


\subsection{Injected bacteria provoke a local interferon type I immune response that can be visualized in transgenic mice by in vivo imaging}

Type-I interferon response is triggered by various host cells and plays critical role during the course of viral or bacterial infections. To investigate interferon response against magnesium-based prolonged $P$. aeruginosa infections, we employed IFN- $\beta$ reporter mice. In these transgenic mice, luciferase is expressed together with IFN- $\beta$ which is a virally and bacterially induced cytokine [41]. Magnesium implants were placed subcutaneously in IFN- $\beta$ reporter mice. Implanted magnesium discs were then infected with wild type P. aeruginosa (PAO1) and the IFN production was observed by in vivo imaging. Side by side, IFN response measured from subcutaneously implanted sterile magnesium and $P$. aeruginosa injected into subcutaneous sham surgical pouches without implants served as controls. Immediately after infection, IFN response was not in detectable limits of the system from the subcutaneous regions in mice carrying infected magnesium, sterile magnesium or infected tissue (Figure 2Ad0, a-c). After one day, robust IFN- $\beta$-luciferase activities were detected from the sites that were infected either with P. aeruginosa alone or in the presence of magnesium implants (Figure 2A, $d 1(a-c))$. IFN-based luminescence was not observed from the sites with sterile magnesium implants during the entire course of experiment (Figure 2A, d1b). In vivo expression of IFN- $\beta$ induced by bacteria administered into sham surgical pouches was detectable for two days and then disappeared. However, robust and prolonged IFN response detectable for two weeks was measured from magnesium infected with $P$. aeruginosa (Figure 2B, filled circles). Moreover, tissue necrosis was observed from these regions (pictures not shown). These results confirm our previous findings that magnesium implants support biofilm formation by $P$. aeruginosa even in reporter mice. 
Prolonged IFN production and resulting tissue necrosis confirm that $P$. aeruginosa biofilms provoke severe localized immune reactions.

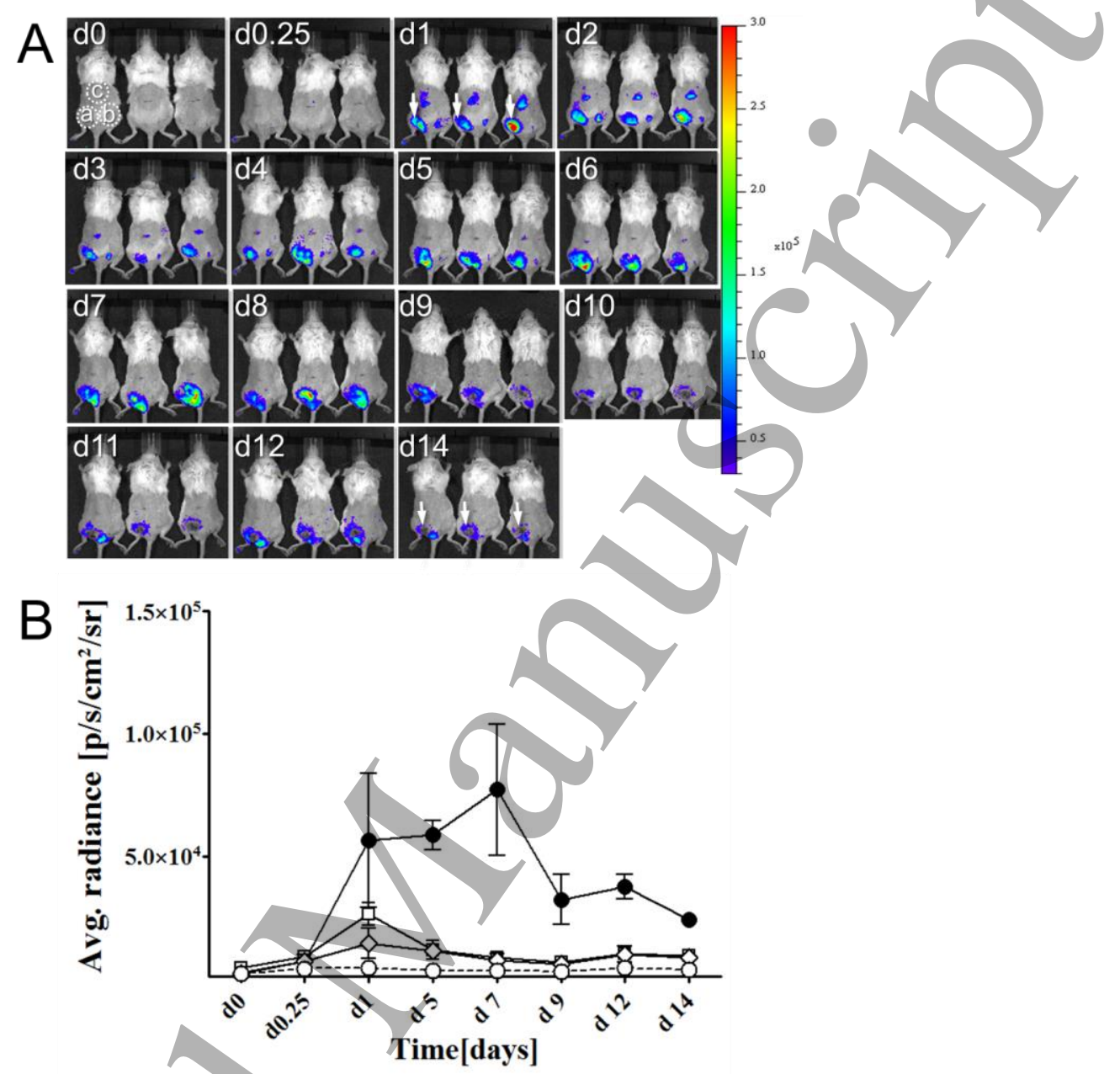

Figure 2: Persistent bacterial infections on implants can be monitored in transgenic mice by in vivo imaging of the host interferon response. Magnesium discs surgically implanted into IFN- $\beta$ reporter mice were infected with wild type P. aeruginosa $(\mathrm{A}, \mathrm{a})$ or kept sterile $(\mathrm{A}, \mathrm{b})$. In parallel, bacteria were injected into sham surgical pouches $(A, C)$. (A). IFN-luciferase activity in response to bacterial infections ( $B$, black circles), in response to injected bacteria in the absence of implants (B, empty squares) or in the presence of sterile magnesium implants (B, empty triangles) and background activity (empty circles) are indicated.

\subsection{P. aeruginosa biofilms cause intense accumulation of host immune cells}

A prolonged IFN response against $P$. aeruginosa biofilms provided evidence that these infections remain restricted at the site of infection. To confirm and to explore the distribution of biofilms, implants after one week of infection were isolated together with adjacent tissue and subjected to electron microscopic analyses. SEM analysis 
revealed $P$. aeruginosa encased in slimy material indicative for biofilms were clearly visible on the surface of implants (Figure3A; asterisk symbols). In parallel, tissue pockets filled with bacteria were visible into the adjacent tissue (Figure 3B; asterisk symbols). Bacterial intrusions into tissue indicate disseminating nature of biofilm infections (Figure 3B). Interestingly, this process was occurring in the presence of large populations of host immune cells (Figure 3C; white arrows). Transmission electron microscopy clearly indicated colonies of $P$. aeruginosa embedding deep into the tissue even in the presence of polymorphonuclear lecukocytes (PMN) (Figure 3D and E; white arrows). Electron transparent regions, a strong indication of biofilms, were clearly visible among bacterial biofilms (Figure 3D; white arrows). P. aeruginosa were observed both intra-and extracellularly in PMN at the site of biofilm formation (Figure 3F; asterisk symbols). This proves that process of biofilm formation was taking place in the presence of active immune cells which were indeed capable of engulfing bacteria around biofilms. Overall, it appeared that bacteria could utilize implant-tissue interfaces to establish biofilms and their distribution was limited to a short distance in the tissue where the spread of the infection was restricted by host immune cells. 
Figure 3: $P$. aeruginosa biofilms persist in the peri-implant tissue even in the presence of host immune system. As described in the legend of figure 1, infected magnesium implants and nearby tissue nourishing biofilms one week post infection were removed, fixed and subjected to electron microscopic analyses. Scanning electron microscopic images of $P$. aeruginosa $\left(^{*}\right.$ ) biofilms present on magnesium implants (A) and in peri-implant tissue (B). Huge influx of host immune cells observed at the site of biofilm formation (C, white arrows). TEM analysis indicates electron transparent regions in the biofilms formed in tissue (D, white arrows) and host PMN around biofilms (E). Bacteria (*) engulfed by host immune cells and present between immune cells (F). Scale bars in each picture $2 \mu \mathrm{m}$.

\subsection{P. aeruginosa biofilms induce strong inflammatory responses in adjacent} tissue

Host inflammatory response against bacterial invasion and foreign material is expected phenomenon $[46,47]$. Contrary to planktonic cultures, biofilms bacteria persist longer in within host tissue even in the presence of active immune system. To evaluate intensity of inflammatory reaction induced by P. aeruginosa biofilms, tissue sections were isolated from mice bearing luminescent $P$. aeruginosa biofilms on magnesium implants after one week of infection and then subjected to hematoxylin and eosin staining. For comparison, tissue sections form sham surgical pouches infected with $P$. 
aeruginsoa and implanted with sterile magnesium were taken as controls. After a week, luminescent $P$. aeruginosa had established biofilms on magnesium implants. Bacteria injected in a sham surgical pouch in the absence of implants disappeared within few days. In an overview, the part of tissue that housed P. aeruginosa biofilms exhibited high degree of inflammation with more obvious infiltration of severe neutrophilic inflammatory infiltrates surrounding the bacterially infected implants (Figure $3 \mathrm{~A}$ and B). Comparatively, tissue portions that were infected with bacteria in the absence of implants or implanted with sterile magnesium elicited mild foreign body reactions (Figure 3C to F). These results strongly indicate that P. aeruginosa biofilms interactions with tissue in the presence of magnesium provoke high degree inflammatory responses.

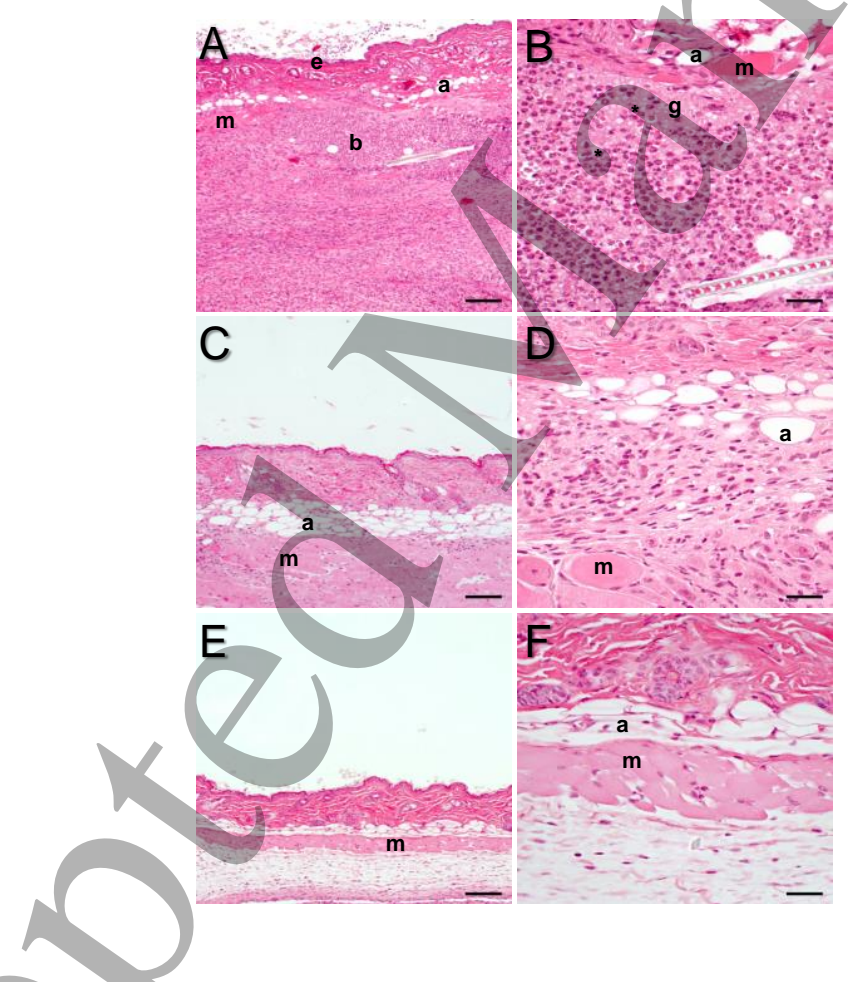

Figure 4: Prolonged local inflammatory reactions triggered in response to $P$. aeruginosa infected implants. As described in the legend of Figure 1, peri-implant tissue was fixed and subjected to H\&E staining. H\&E stained tissue sections bearing biofilms on implant surfaces (A and B), infected with bacteria after sham surgery (C and D) and tissue bearing sterile magnesium implants ( $E$ and F). Scale bars on the left side correspond to $100 \mu \mathrm{m}$, scale bars on the right side to $26 \mu \mathrm{m}$. Labeled structures: epidermis (e); adipose cells (a); muscle fibers (m); bacteria (b); granular tissue (g); host immune cells $\left(^{*}\right)$.

\subsection{Evidence of biofilm constituents in peri-implant tissue}


Biofilm matrix is mainly composed from sugar polymers and proteins that can be visualized by periodic acid-Schiff (PAS) staining [48, 49]. Even though, electron microscopic analyses clearly revealed P. aeruginosa biofilms in tissue however PAS staining was applied to further verify $P$. aeruginosa biofilms in the tissue near infected magnesium implants. As expected, PAS positive material indicative for the presence of biofilms matrix was detected in tissue harboring infected magnesium implants (Figure 4A and B, black arrow). While, no PAS positive material could be detected hear infected areas in the absence of implants or in tissue with sterile magnesium implants (Figure 4C to F). The data also indicated that bacterial implant infections were not solely restricted to implant surface. Instead, they expanded a short distance into the tissue and were walled off by host immune cells.

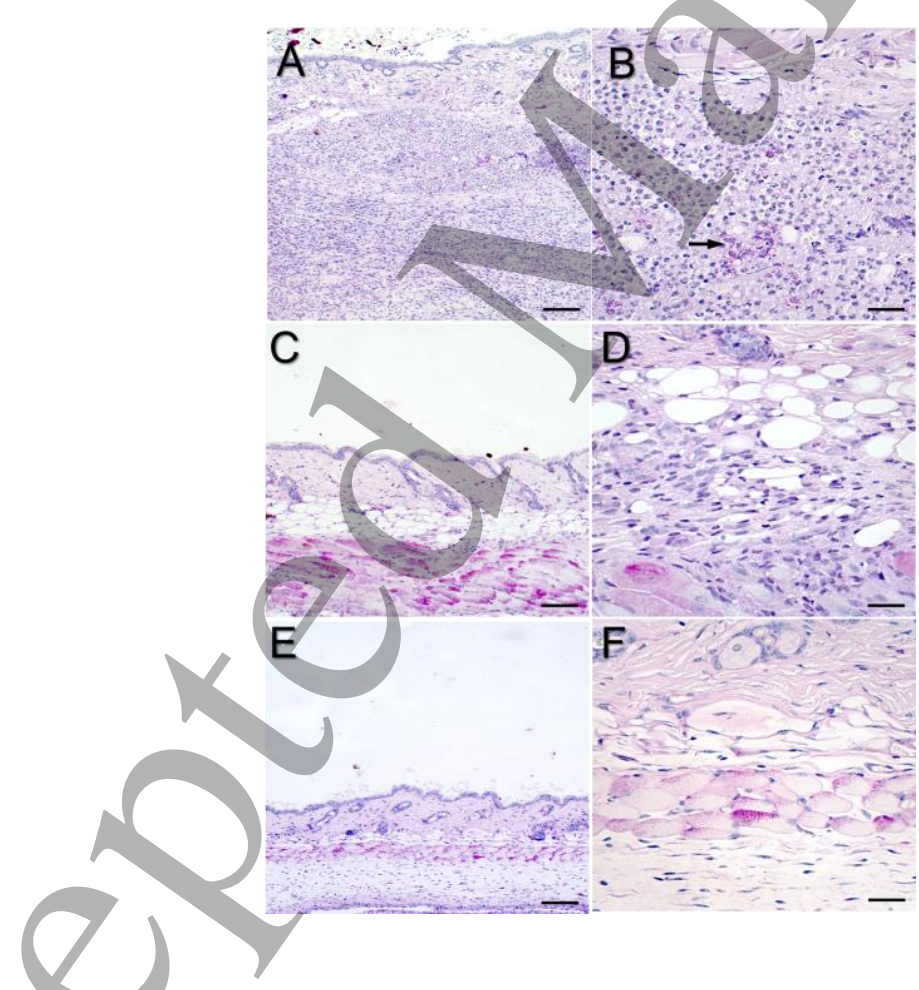

Figure 5: Evidence for biofilm matrix material (ECM) on infected magnesium implants extending a short range into peri-implant tissue. The tissue was fixed and subjected to PAS staining for histological examination by light microscopy. PAS positive staining of $P$. aeruginosa in peri-implant tissue $(\mathrm{A}$ and $\mathrm{B}$ black arrow) propagated from infected magnesium implants, PAS stained tissue infected with bacteria in the absence of implants ( $C$ and D) and tissue bearing sterile magnesium implants ( $E$ and F). Scale bars on the left side correspond to $100 \mu \mathrm{m}$, scale bars on the right side to $26 \mu \mathrm{m}$

\subsection{Histological evidence for biofilm invasion into peri-implant tissue}


Previous analyses confirm that tissues near infected implants are filled with $P$. aeruginosa biofilms. To identify bacteria in tissue, antibodies targeting $P$. aeruginosa were applied on the tissue sections isolated from the vicinity of infected magnesium. Interestingly, a distinct region positive for pseudomonas staining was visible in the tissue (Figure 5A, grey area marked by a black arrow). At high magnification, it could be seen that $P$. aeruginosa were dispersed within this particular area of biofilm formation (Figure 5B, black arrow pointing $P$. aeruginosa). The area positive for pseudomonas staining was surrounded by high influx of inflammatory cells which confirms biofilm resistance towards host immune cells (Figure 5B, black arrow). Tissue bearing sterile magnesium or infected with bacteria alone were negative for pseudomonas staining (Figure 5C to F). Overall, various tissue staining techniques confirmed that $P$. aeruginosa biofilms were not limited to the implant surface instead they penetrate into the nearby tissue and trigger the induction of strong immune reactions (Table 1).

\section{Table 1}

Inflammation intensity in tissue adjacent to biofilm bearing magnesium discs, magnesium alone and infection alone.

\begin{tabular}{|c|c|c|c|}
\hline Tissue samples & $\begin{array}{c}\text { H\&E } \\
\text { staining }\end{array}$ & PAS staining & $\begin{array}{c}\text { Pseudomonas } \\
\text { staining }\end{array}$ \\
\hline Bacterially infected magnesium implants & $4^{*}$ & 3 & 3 \\
\hline Bacterial infection without implants & 0 & 0 & 0 \\
\hline Sterile implants & 1 & 0 & 0 \\
\hline
\end{tabular}

Numbers indicate an arbitrary inflammatory score from 0 (no response observed in any of three samples) to 4 (distinct inflammatory response in all three samples investigated). The data shown were compiled by an extensive analysis by an experienced histologist. 


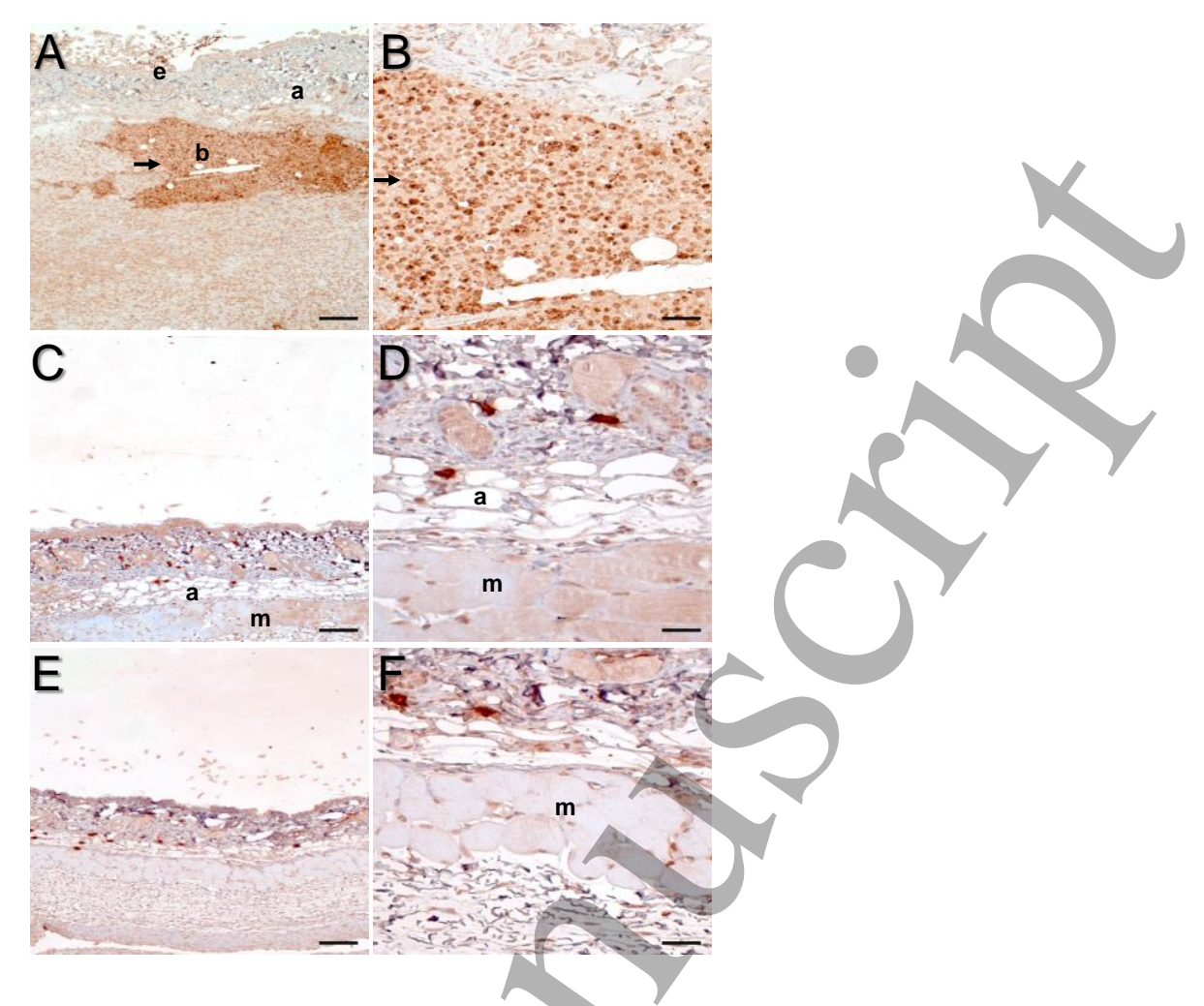

Figure 6: $P$. aeruginosa infections originating from medical implant surfaces extend into the adjacent tissue. As described in the legend of Figure 1, peri-implant tissue was fixed and subjected to $P$. aeruginosa-specific immuno staining. $P$. aeruginosa biofilms in peri-implant tissue (A and B black arrows), immuno-stained tissue infected with bacteria in the/absence of implants ( $C$ and D) and tissue bearing sterile magnesium implants ( $\mathrm{E}$ and $\mathrm{F}$ ). Labeled structures are same as described in the legend of Figure 3. Black arrows indicate regions with evidence for biofilm formation.

\subsection{Splenomegaly in mice with P. aeruginosa infected magnesium implants}

Strong immunogenic and tissue inflammatory reactions associated with the presence of magnesium based $P$. aeruginosa biofilms convince that these infections are not localized and can spread into adjacent tissue. In the next step, systemic effects of biofilms were investigated by observing liver, spleen and gut of mice bearing P. aeruginosa biofilms. These organs were then homogenized and number of bacteria was determined. Bacterial colony forming units could not be counted from liver or spleen except intestine which seemed normal flora (Table 2). Morphology of intestine and liver of mice bearing $P$. aeruginosa biofilms was normal and similar to un-infected animals. Surprisingly, size as well as weight of spleen isolated from infected mice had increased 
compared to un-infected mice (Figure 7A compare a with b). These results provide evidence that biomaterial-associated biofilm infections in host affect systemic organs, particularly spleen. Importantly, such significant increase in the size and weight of spleen are indicative of additional complications for patients suffering from biomaterial-associated infections.
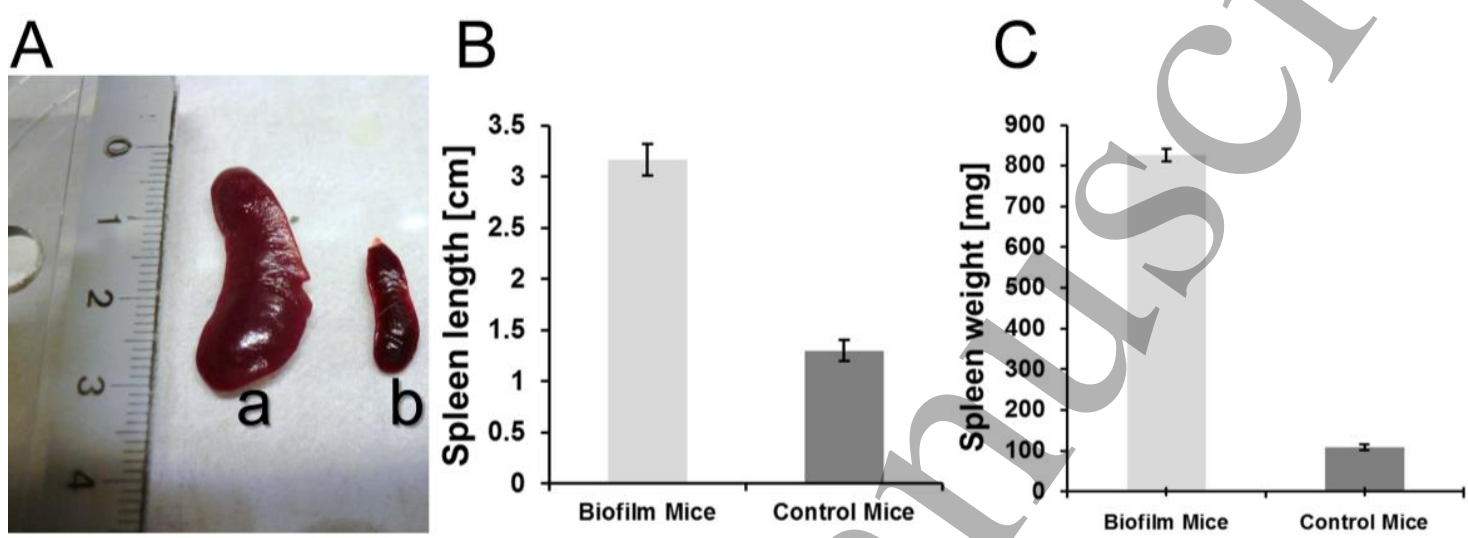

Figure 7: Prolonged $P$. aeruginosa biofilms on magnesium implants lead to significant increase in the size of spleen. Magnesium implants were infected with growing cultures of $P$. aeruginosa or kept uninfected in individual mice. After 8 days, mice were euthanized and their organs were removed and imaged with a light microscope (A). Spleen of mice bearing biofilm (A, a) and spleen of control mice (A, b). Changes in length (B) and weight (C) of spleen isolated from mice bearing infected or sterile magnesium implants.

\section{Table 2}

Bacterial colony forming units (CFUs) obtained from various tissue samples derived from mice carrying subcutaneous $P$. aeruginosa infected magnesium implants

\begin{tabular}{ccccc}
\hline $\begin{array}{c}\text { Time post } \\
\text { infection }\end{array}$ & $\begin{array}{c}\text { CFUs/magnesium } \\
\text { implant }\end{array}$ & $\begin{array}{c}\text { CFUs/g peri- } \\
\text { implant tissue }\end{array}$ & CFUs/g gut tissue & $\begin{array}{c}\text { CFUs/gram } \\
\text { of liver }\end{array}$ \\
\hline \multirow{2}{*}{1 week } & $3.60^{9} \pm 1.51 \mathrm{e} 9$ & $2.77^{9} \pm 1.84 \mathrm{e} 9$ & $2.73^{4} \pm 1527$ & 0 \\
\hline
\end{tabular}

\section{Discussion}

The results in this study confirm and refine previous results in that in vitro antibacterial properties of pure magnesium are not sufficient to prevent bacterial colonization of 
implants and instead apparently even promote prolonged bacterial survival under in vivo situations. We and some other studies have previously reported that in vitro antibacterial properties of magnesium are due to high alkalization therefore one explanation for the absence of in vivo antimicrobial properties could therefore be the absence of high $\mathrm{pH}$ in the peri-implant tissue controlled by the efficient body buffering system [26, 50]. In addition, development of conditioning film by blood plasma or wound liquid creates a barrier for the dissemination of magnesium corrosion products thereby allowing colonization of bacteria $[51,52]$. Since, hydrogen gas is degradation product of magnesium corrosion, it cannot be excluded that the formation of gas pockets on magnesium surface provides protection to bacteria from early host cells invasion and subsequently develop biofilms. Therefore, before clinical applications can be envisioned, it will be necessary to enhance antibacterial properties of magnesium. Since magnesium alloys can be manufactured that have superior mechanical qualities, it could be envisioned to add antibacterial acting metal alloys, such as copper or silver [53, 54]. Since in this study no spontaneous infections of magnesium alloy implants were observed, it may be sufficient to provide a temporary protection for a limited time after surgery by antibiotic-releasing coatings until the wound has closed and the implant is protected by a fibrous capsule [55]. By the application of these strategies susceptibility of magnesium implants towards biofilm infections could be reduced and it can be promoted for clinical applications.

Pseudomonas aeruginosa is significant pathogen responsible for implant failure in patients due to its biofilms. One important question about $P$. aeruginosa biofilms is whether bacteria are localized on implant surface, in the interface zone or evade the tissue. Secondly, there is limited information about host immune responses against 
these biofilms. To answer these questions, the major challenge is lack of clinically relevant animal models. Previous studies report that bacterial biofilms are mainly restricted on or near the surfaces of implant materials because mechanical and structural properties of these materials which protect them from being engulfed by the host immune cells [56-58]. Whereas, a recent study reported that $S$. epidermidis biofilms penetrated into the nearby tissues after initially growing on implanted materials resulting in robust inflammatory immune reactions [35]. However, in depth characterization of biofilms-mediated immune responses were not done in any of these studies. Here, we report for the first time biofilm mediated immune responses in a highly sensitive reporter mouse expressing type I interferon luciferase under transcriptional control of the IFN- $\beta$ promoter [41]. Our results indicate robust and persistent type-I IFN induction from $P$. aeruginosa biofilms s as compared to controls. IFN responses against $P$. aeruginosa biofilms were not immediate and were visible after 24 hours of infection. Though molecular mechanisms for delayed IFN response were not investigated however an explanation could be that initially injected planktonic bacteria were not sufficient to stimulate IFN production. While at later stages, bacterial number increased by biofilm formation resulting in IFN production. It also confirms that $P$. aeruginosa biofilms are recalcitrant against immune system even in the presence of IFN secreting active host cells. Additionally, we restricted our research to the finding that strong IFN production is induced in response to $P$. aeruginosa biofilms. However, molecular mechanisms responsible for the activation of IFN in this mouse model need to be investigated. Polymorphonuclear lecukocytes (PMNs) are powerful biocidal innate immune cells that can attack and occasionally even eliminate biomaterial-associated biofilms. Formation of P. aeruginosa biofilms in tissue together with PMNs indicates that implanted material provides initial niches for planktonic bacteria which may otherwise 
be easily captured and killed by these cells in the absence of magnesium implants. At later stages, bacteria switch to biofilms and are strong enough to cope with immune cells. Resultantly, bacterial biofilms from implant surface penetrate into the nearby tissue and persist there as well. Detection of inflammatory cells, bacteria and biofilm matrix components in the same tissue endorses that biofilms migrate from implanted surface to adjacent tissue and are certainly resistant towards host immune system [5961]. Biofilms in the tissue provides compelling evidences for proliferating nature of these infections involving strong inflammatory reactions. Such high influx of immune cells against biofilms could have involvement of systemic organs therefore various body organs of the mice with biofilm overgrown implants were observed. Interestingly, no systemic organ infiltration by bacteria could be detected by using the approaches described in this study however splenomegaly in mice with biofilms was observed. Splenomegaly is reported due to various reasons but it is the first time that we observed it in animal suffering from biofilm infections $[62,63]$. It is possible that cytokines or migratory immune cells originating at the infected region could affect the inflammatory spleen response. Increases in the spleen size could nevertheless be indicative of systemic bacterial spread, perhaps at a level too low to detect by the methods applied here or it could be caused by the systemic distribution of inflammatory bacterial degradation products.

The present study validated both localized and systemic inflammatory immune responses to bacterial biofilms established on magnesium implant surfaces. Thus, novel strategies need to be established to control such infections.

\section{Conclusion}




\section{Acknowledgement}

We are thankful to Prof. Dr. Peter Paul Mueller for his guidance and valuable suggestions in the preparation of this manuscript. We thank Susanne Häussler and Siegfried Weiss for donating bacterial strains and transgenic mice and Ina Schleicher for technical assistance in the electron microscopic studies. We are thankful to Rainer Eifler and Jan-Martin Seitz from Institute of Materials Science, Leibniz University of Hannover for the provision of magnesium implants. We gratefully acknowledge the funding of this work by the German Research Foundation (DFG) within the Collaborative Research Centre (SFB 599), Higher Education Commission (HEC), Pakistan and the German Academic Exchange Service (DAAD), Germany.

\section{References}

[1] Chen Q, Thouas GA. Metallic implant biomaterials. Materials Science and Engineering: R: Reports. 2015;87:1-57.

[2] Oliver A, Cantón R, Campo P, Baquero F, Blázquez J. High Frequency of Hypermutable Pseudomonas aeruginosa in Cystic Fibrosis Lung Infection. Science. 2000;288:1251-3.

[3] Rasamiravaka T, Labtani Q, Duez P, El Jaziri M. The Formation of Biofilms by Pseudomonas aeruginosa: A Review of the Natural and Synthetic Compounds Interfering with Control Mechanisms. BioMed Research International. 2015;2015:17.

[4] Hall-Stoodley L, Costerton JW, Stoodley P. Bacterial biofilms: from the Natural environment to infectious diseases. Nat Rev Micro. 2004;2:95-108.

[5] Joo H-S, Otto M. Molecular Basis of In Vivo Biofilm Formation by Bacterial Pathogens. Chemistry \& Biology. 2012;19:1503-13. 
[6] Zapotoczna M, O'Neill E, O'Gara JP. Untangling the Diverse and Redundant Mechanisms of Staphylococcus aureus Biofilm Formation. PLoS Pathog. 2016;12:e1005671.

[7] Wu H, Moser C, Wang H-Z, Hoiby N, Song Z-J. Strategies for combating bacterial biofilm infections. In J Oral Sci. 2015;7:1-7.

[8] Arciola CR, Campoccia D, Speziale P, Montanaro L, Costerton JW. Biofilm formation in Staphylococcus implant infections. A review of molecular mechanisms and implications for biofilm-resistant materials. Biomaterials. 2012;33:5967-82.

[9] Zhao B, van der Mei HC, Subbiahdoss G, de Vries J, Rustema-Abbing M, Kuijer R, et al. Soft tissue integration versus early biofilm formation on different dental implant materials. Dental Materials. 2014;30:716-27.

[10] Hooper LV, Littman DR, Macpherson AJ. Interactions Between the Microbiota and the Immune System. Science. 2012;336:1268-73.

[11] McNab F, Mayer-Barber K, Sher A, Wack A, O'Garra A. Type I interferons in infectious disease. Nat Rev Immunol. 2015;15:87-103.

[12] Hänsch GM. Host Defence against Bacterial Biofilms: Mission Impossibe? ISRN Immunology. 2012;2012:17.

[13] Fowler VG, Jr, Allen KB, Moreira ED, et al. Effect of an investigational vaccine for preventing staphylococcus aureus infections after cardiothoracic surgery: A randomized trial. JAMA. 2013;309:1368-78.

[14] Uçkay I, Hoffmeyer P, Lew D, Pittet D. Prevention of surgical site infections in orthopaedic surgery and bone trauma: state-of-the-art update. Journal of Hospital Infection. 2013;84:5-12.

[15] Puckett SD, Taylor E, Raimondo T, Webster TJ. The relationship between the nanostructure of titanium surfaces and bacterial attachment. Biomaterials. 2010;31:706-13.

[16] Neoh KG, Hu X, Zheng D, Kang ET. Balancing osteoblast functions and bacterial adhesion on functionalized titanium surfaces. Biomaterials. 2012;33:2813-22.

[17] Zimmerli W, Moser C. Pathogenesis and treatment concepts of orthopaedic biofilm infections. FEMS Immunology \&amp; Medical Microbiology. 2012;65:158-68.

[18] Sanclement JA, Webster P, Thomas J, Ramadan HH. Bacterial Biofilms in Surgical Specimens of Patients with Chronic Rhinosinusitis. The Laryngoscope. 2005;115:578-82.

[19] Bjarnsholt T, Alhede M, Alhede M, Eickhardt-Sørensen SR, Moser C, Kühl M, et al. The in vivo biofilm. Trends in Microbiology.21:466-74.

[20] Patel SS, Aruni W, Inceoglu S, Akpolat YT, Botimer GD, Cheng WK, et al. A comparison of Staphylococcus aureus biofilm formation on cobalt-chrome and titanium-alloy spinal implants. Journal of Clinical Neuroscience. 2016;31:219-23.

[21] Davies D. Understanding biofilm resistance to antibacterial agents. Nat Rev Drug Discov. 2003;2:114-22.

[22] Lindsay D, von Holy A. Bacterial biofilms within the clinical setting: what healthcare professionals should know. Journal of Hospital Infection. 2006;64:313-25.

[23] Goodman SB, Yao Z, Keeney M, Yang F. The future of biologic coatings for orthopaedic implants. Biomaterials. 2013;34:3174-83.

[24] Raphel J, Holodniy M, Goodman SB, Heilshorn SC. Multifunctional coatings to simultaneously promote osseointegration and prevent infection of orthopaedic implants. Biomaterials.

2016;84:301-14.

[25] Li Y, Liu L, Wan P, Zhai Z, Mao Z, Ouyang Z, et al. Biodegradable Mg-Cu alloy implants with antibacterial activity for the treatment of osteomyelitis: In vitro and in vivo evaluations.

Biomaterials. 2016;106:250-63.

[26] Rahim MI, Eifler R, Rais B, Mueller PP. Alkalization is responsible for antibacterial effects of corroding magnesium. Journal of Biomedical Materials Research Part A. 2015;103:3526-32.

[27] Tie D, Feyerabend F, Mueller W-D, Schade R, Liefeith K, Kainer KU, et al. Antibacterial biodegradable Mg-Ag alloys. European cells \& materials. 2012;25:284-98; discussion 98. 
[28] Rahim MI, Weizbauer A, Evertz F, Hoffmann A, Rohde M, Glasmacher B, et al. Differential magnesium implant corrosion coat formation and contribution to bone bonding. Journal of Biomedical Materials Research Part A. 2017;105:697-709.

[29] Tan L, Yu X, Wan P, Yang K. Biodegradable Materials for Bone Repairs: A Review. Journal of Materials Science \& Technology. 2013;29:503-13.

[30] Castellani C, Lindtner RA, Hausbrandt P, Tschegg E, Stanzl-Tschegg SE, Zanoni G, et al. Boneimplant interface strength and osseointegration: Biodegradable magnesium alloy versus standard titanium control. Acta Biomaterialia. 2011;7:432-40.

[31] Windhagen H, Radtke K, Weizbauer A, Diekmann J, Noll Y, Kreimeyer U, et al. Biodegradable magnesium-based screw clinically equivalent to titanium screw in hallux valgus surgery: short term results of the first prospective, randomized, controlled clinical pilot study. BioMedical) Engineering OnLine. 2013;12:62.

[32] Rahim MI, Rohde M, Rais B, Seitz J-M, Mueller PP. Susceptibility of metallic magnesium implants to bacterial biofilm infections. Journal of Biomedical Materials Research Part A. 2016;104:1489-99.

[33] Brooks EK, Ahn R, Tobias ME, Hansen LA, Luke-Marshall NR, Wild L, et al. Magnesium alloy AZ91 exhibits antimicrobial properties in vitro but not in vivo. Journal of Biomedical Materials Research Part B: Applied Biomaterials. 2017:n/a-n/a.

[34] Peng H, Changli Z, Pengfei C, Hongliu W, Jiahua N, Shaoxiang Z, et al. Reduced antibacterial property of metallic magnesium in vivo. Biomedical Materials. 2017;12:015010.

[35] Broekhuizen CAN, de Boer L, Schipper K, Jones CD, Quadir S, Feldman RG, et al. Peri-Implant Tissue Is an Important Niche for Staphylococcus epidermidis in Experimental BiomaterialAssociated Infection in Mice. Infection and Immunity. 2007;75:1129-36.

[36] Dhir S. Biofilm and dental implant: The microbial link. Journal of Indian Society of Periodontology. 2013;17:5-11.

[37] Blow N. In vivo molecular imaging: the inside job. Nat Meth. 2009;6:465-9.

[38] Rahim MI, Rohde M, Rais B, Seitz J-M, Mueller PP. Susceptibility of metallic magnesium implants to bacterial biofilm infections. Journal of Biomedical Materials Research Part A.

2016:n/a-n/a.

[39] Rahim MI, Eifler R, Rais B, Mueller PP. Alkalization is responsible for antibacterial effects of corroding magnesium. Journal of biomedical materials research Part A. 2015;103:3526-32.

[40] Badar M, Rahim MI, Kieke M, Ebel T, Rohde M, Hauser H, et al. Controlled drug release from antibiotic-loaded layered double hydroxide coatings on porous titanium implants in a mouse model. Journal of biomedical materials research Part A. 2015;103:2141-9.

[41] Lienenklaus S, Cornitescu M, Ziętara N, tyszkiewicz M, Gekara N, Jabłońska J, et al. Novel Reporter Mouse Reveals Constitutive and Inflammatory Expression of IFN- $\beta$ In Vivo. The Journal of Immunology. 2009;183:3229-36.

[42] Pawar V, Komor U, Kasnitz N, Bielecki P, Pils MC, Gocht B, et al. In Vivo Efficacy of Antimicrobials against Biofilm-Producing Pseudomonas aeruginosa. Antimicrobial Agents and Chemotherapy. 2015;59:4974-81.

[43] Komor U, Bielecki P, Loessner H, Rohde M, Wolf K, Westphal K, et al. Biofilm formation by Pseudomonas aeruginosa in solid murine tumors - a novel model system. Microbes and Infection. 2012;14:951-8.

[44] Spurr AR. A low-viscosity epoxy resin embedding medium for electron microscopy. Journal of ultrastructure research. 1969;26:31-43.

[45] Rais B, Köster M, Rahim MI, Pils M, Seitz J-M, Hauser H, et al. Evaluation of the inflammatory potential of implant materials in a mouse model by bioluminescent imaging of intravenously injected bone marrow cells. Journal of Biomedical Materials Research Part A. 2016;104:2149-58. [46] Medzhitov R. Recognition of microorganisms and activation of the immune response. Nature. 2007;449:819-26. 
[47] Veiseh O, Doloff JC, Ma M, Vegas AJ, Tam HH, Bader AR, et al. Size- and shape-dependent foreign body immune response to materials implanted in rodents and non-human primates. Nat Mater. 2015;14:643-51.

[48] Donlan RM. Biofilms and device-associated infections. Emerging Infectious Diseases. 2001;7:277-81.

[49] Anderson JM, Rodriguez A, Chang DT. Foreign body reaction to biomaterials. Seminars in immunology. 2008;20:86-100.

[50] Qin H, Zhao Y, Cheng M, Wang Q, Wang Q, Wang J, et al. Anti-biofilm properties of magnesium metal via alkaline pH. RSC Advances. 2015;5:21434-44.

[51] Wagner C, Aytac S, Hänsch GM. Biofilm growth on implants: bacteria prefer plasma coats. The International journal of artificial organs. 2011;34:811-7.

[52] Charyeva O, Neilands J, Svensäter G, Wennerberg A. Bacterial biofilm formation on resorbing magnesium implants. Open Journal of Medical Microbiology. 2015;5:1.

[53] Tie D, Feyerabend F, Müller WD, Schade R, Liefeith K, Kainer KU, et al. Antibacterial biodegradable Mg-Ag alloys. European cells \& materials2013. p. 284-98; discussion 98.

[54] Jähn K, Saito H, Taipaleenmäki H, Gasser A, Hort N, Feyerabend F, et al. Intramedullary Mg2Ag nails augment callus formation during fracture healing in mice. Acta Biomaterialia. 2016;36:35060.

[55] Hesse D, Badar M, Bleich A, Smoczek A, Glage S, Kieke M, et al. Layered double hydroxides as efficient drug delivery system of ciprofloxacin in the middle ear: an animal study in rabbits. Journal of Materials Science: Materials in Medicine. 2013;24:129-36.

[56] McConoughey SJ, Howlin R, Granger JF, Manring MM, Calhoun JH, Shirtliff M, et al. Biofilms in periprosthetic orthopedic infections. Future Microbiology. 2014;9:987-1007.

[57] Windolf CD, Meng W, Logters TT, MacKenzie CR, Windolf J, Flohe S. Implant-associated localized osteitis in murine femur fracture by biofilm forming Staphylococcus aureus: a novel experimental model. Journal of orthopaedic research : official publication of the Orthopaedic Research Society. 2013;31:2013-20.

[58] Flores-Mireles AL, Walker JN, Caparon M, Hultgren SJ. Urinary tract infections: epidemiology, mechanisms of infection and treatment options. Nat Rev Micro. 2015;13:269-84.

[59] Jensen PO, Givskov M, Bjarnsholt T, Moser C. The immune system vs. Pseudomonas aeruginosa biofilms. FEMS immunology and medical microbiology. 2010;59:292-305.

[60] Valle J, Solano C, García B, Toledo-Arana A, Lasa I. Biofilm switch and immune response determinants at early stages of infection. Trends in Microbiology. 2013;21:364-71.

[61] Monack DM, Hultgren SJ. The complex interactions of bacterial pathogens and host defenses. Current opinion in microbiology. 2013;16:1-3.

[62] Motyckova G, Steensma DP. Why Does My Patient Have Lymphadenopathy or Splenomegaly? Hematology/Oncology Clinics of North America. 2012;26:395-408.

[63] Kang GH, Moon HS, Lee ES, Kim SH, Sung JK, Lee BS, et al. A Case of Liver Fibrosis with Splenomegaly after Oxaliplatin-Based Adjuvant Chemotherapy for Colon Cancer. J Korean Med Sci. 2013;28:1835-8. 